\title{
Research on the Optimization Design of Propeller Considered the Off-designed Conditions
}

\author{
Shuai Sun, Xin Chang, Chao Wang, Tao Mo \\ College of Shipbuilding Engineering, Harbin Engineering University, Harbin, China \\ Email: sunshuaidoc@163.com
}

Keywords: propeller optimization; off-design conditions; simple genetic algorithm.

\begin{abstract}
In order to improve the performance of propeller, the propeller efficiency optimization method under the off-designed conditions were studied. Taking the open water efficiency around the designed velocity of parent propeller as the objective function, taking the radial distribution of pitch ratio as the optimization variables and taking the thrust and the cavitation performance of the propeller as the constraints and the optimization mathematical model was established. The panel method was used to solve the hydrodynamic performance of propeller and the cavitation performance was described by the BaiLiEr merchant margin line. Based on the simple genetic algorithm, the optimized radial distribution of the pitch ratio was solved. The optimized results show that due to the changes of ship speed the method which takes the efficiency of design velocity as the objective function fails to achieve the expected energy-saving. And the multiple operating points corresponding efficiency around designed speed was supposed to considered.
\end{abstract}

\section{Introduction}

The design of effective ship propeller has been the key problem on the research of ship hydrodynamics. Currently, to obtain the maximum efficiency design and optimization of ship propeller are usually carried on under the design speed. The designers take the existing mature theory or optimization algorithm to optimize the design of the propeller considering one or more elements of propeller, such as optimizing the distribution of pitch, the blade profile shape optimization [1,5] etc.

However, the practice shows that simply considering the design speed, the optimization design of propeller often falls flat in the process of actual operation. The reason is that due to wind, wave, streams and changes in ship displacement, the advance coefficient of propeller cannot be maintained at design conditions. As a result the propeller cannot always work at optimal efficiency.

Thus, further optimization of propeller design is necessary. This requires propeller efficiency optimal within a certain range of design advance coefficient, and taking it as the optimization objective function to optimize the design of propeller.

The simple genetic algorithm combined with the weighted sum method is applied for analyzing these problems, and the propeller optimization design methods considering non-design conditions are initially established in this paper.

\section{Propeller optimization mathematical model}

\section{Optimization objective function}

The open water efficiency curve for a parent type propeller is shown as Fig.1, and the corresponding advance coefficient to optimal efficiency is $J_{0}$. When advance coefficient is $J_{0}$, the corresponding propeller open water efficiency is regarded as the optimization objective function by the traditional optimization design method.

When considering the fluctuation of advance coefficient during the actual operation of the ship, its fluctuation range is the neighborhood of $J_{0}$. Assuming the neighborhood radius is $\Delta$, the optimization design in this paper is required to maximize the open water efficiency $\eta_{o}$ within the range of $U\left(J_{o}, \Delta\right)=\left\{J \mid J_{o}-\Delta<J<J_{o}+\Delta\right\}$. N discrete points with uniform distribution are selected 
in the neighborhood of $U\left(J_{o}, \Delta\right)$ during actual processing. The discrete points are listed like $J_{i}, i=1,2, \cdots, N$ (including the boundary points $J_{A}$ and $J_{B}$, and the $N$ is odd, to guarantee $J_{0}$ is also the optimization target), then

$$
\begin{aligned}
& J 1=J 0-\triangle \\
& J_{N}=J_{0}+\Delta
\end{aligned}
$$

(1)

$$
J_{i}=J_{1}+2 i \Delta /(N-1), i=2,3, \ldots, N-1
$$

Thus, both the optimal efficiency $\eta_{o}\left(J_{o}\right)$ considering point $J_{0}$ and efficiency $\eta_{O}\left(J_{i}\right), i \in[1, N], i \neq(N+1) / 2$ in the neighborhood of $U\left(J_{o}, \Delta\right)$ compose the optimization objective function.

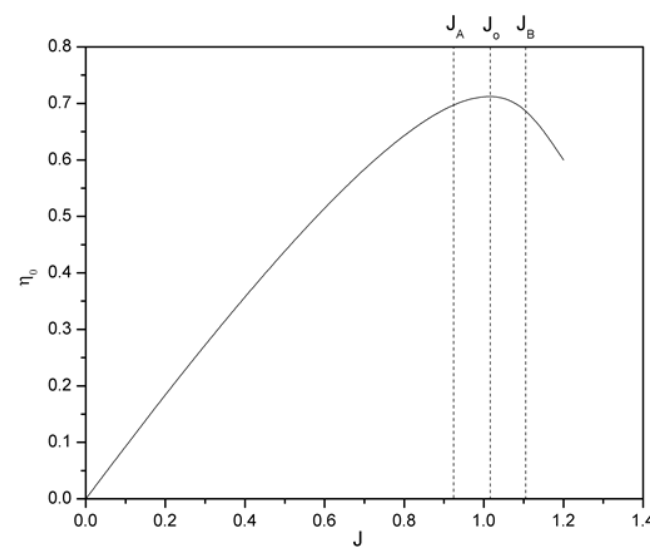

Fig.1 Open water efficiency of propeller

\section{The optimization design variables}

In this paper, radial pitch ratio distribution $P(R) / D$ is regarded as the optimization design variable. In order to the uniform distribution of the optimized pitch ratio along the radial direction, the pitch ratio distribution curve along the radial is described by the linear perturbation method based on Hicks-Henne shape function [6] shown in equation (2).

$$
\begin{aligned}
& r=r_{0} \\
& P(r) / D=P(r)_{O} / D+\sum_{i} a_{i} f_{i} \\
& f_{k}(x)=\sin ^{3}\left(\pi\left(\frac{x-d / D}{1-d / D}\right)^{\lg 0.5 / \operatorname{gg} x_{k}}\right) \quad k=1, n
\end{aligned}
$$

Wherein, $d / D$ is the hub diameter ratio. $x_{k}$ is uniformly selected from $x=d / D \sim 1.0$. For a radial distribution curves of pitch $k=6$ is available. The optimization variables are six control parameters of the pitch distribution curve. The form of the control parameters is like $a_{1}, a_{2}, \cdots, a_{6}$.

The constraints are to meet the propeller cavitation performance and strength requirement. The strength requirement just ensure that the blade thickness is not less than the parent paddle, and propeller cavitation performance is forecast by the use of Bo-Lier merchant Limit Line [7].

Therefore, a mathematical model of optimum design of the propeller can be expressed as equation (3) in this paper,

Where, objective function $f_{i}$ is solved by equation $\eta_{o}=\frac{T V_{A}}{2 \pi n Q}$.

$$
\begin{array}{cl}
\max & f_{i}=\eta_{o}\left(J_{i}\right)=f_{i}\left(a_{1}, a_{2}, \cdots, a_{6}\right), i=1,2, \cdots, N \\
\text { s.t. } & g_{1}\left(a_{1}, a_{2}, \cdots, a_{6}\right)=\tau_{c}-\tau_{c}{ }^{\prime}<0 \\
& g_{2}\left(a_{1}, a_{2}, \cdots, a_{6}\right)=K_{T}-K_{T o}>0 \\
& g_{3}\left(a_{1}, a_{2}, \cdots, a_{6}\right)=K_{Q}-K_{Q o} \leq 0 \\
& a_{1}, a_{2}, \cdots, a_{6} \geq-0.004 \\
& a_{1}, a_{2}, \cdots, a_{6} \leq 0.004
\end{array}
$$




\section{The constraint conditions}

The $g_{1}$ is cavitation constraints. The $g_{2}$ is trust constraints and $g_{3}$ is torque constraints. In the equation (3), $K_{T}=\frac{T}{\rho n^{2} D^{4}}, K_{Q}=\frac{T}{\rho n^{2} D^{5}}, K_{T o}$ and $K_{Q o}$ is respectively the trust coefficient and torque coefficient of parent propeller.

\section{Determination of the objective function}

The variation of velocity within a certain range should be considered in this problem. Where the $J_{i}$ and $J_{0}$ respectively correspond to the actual speed $V_{S}$ and the design speed $V_{0}$.

Due to the voyage time with different speed is different, so the efficiency under different speed contribution to its performance is different too. Therefore, the corresponding weights $\omega_{k}$ on the efficiency of different speed can be set and of the objective function is multiplied by the corresponding weight value $\omega_{k}$, and then add them up to construct a new function $z(x)$. Thus the optimization problem of multiple objective functions into a single objective function $z(x)$ optimization problem. As a result, the optimization problem of multiple objective functions $f_{i}$ will be transformed into a single objective function $z(x)$ optimization problem.

Assuming ship speed curve over time is shown in Fig. 2. Measured once every interval $t$, the total measurement time is $T$. In the time range $T$ within the variation range of true speed $V_{S}(t)$ is $R_{V} \subseteq\left\{V_{S}(t) \mid V_{S}(t) \in\left[V_{A}, V_{B}\right]\right\}$ which is a set of $\left[V_{A}, V_{B}\right]$.

The set $R_{V}$ is uniformly divided into $N$ subsets $r_{i}, i=1, \cdots, N$, then obviously the midpoint of each subset is the speed $V_{r i}$ corresponding with $J_{i}$, namely equation (4) set up.

In particular the midpoint of $r_{1}$ is $V_{A}$, the midpoint of $r_{N}$ is $V_{B}$, the midpoint of $r_{\frac{N+1}{2}}$ is $V_{0}$ which is shown in Fig.3. Consequently, the weight $w_{i}$ values of the objective function corresponding to $J_{i}$ can be obtained according to the statistical results.

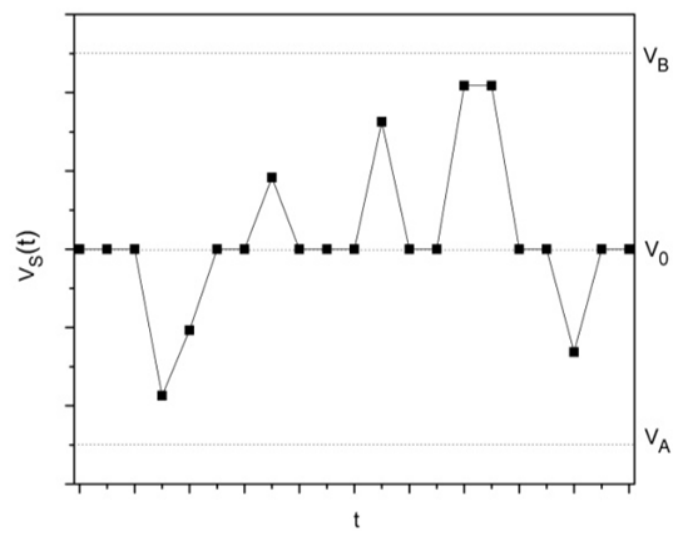

Fig.2 The full-scale ship speed

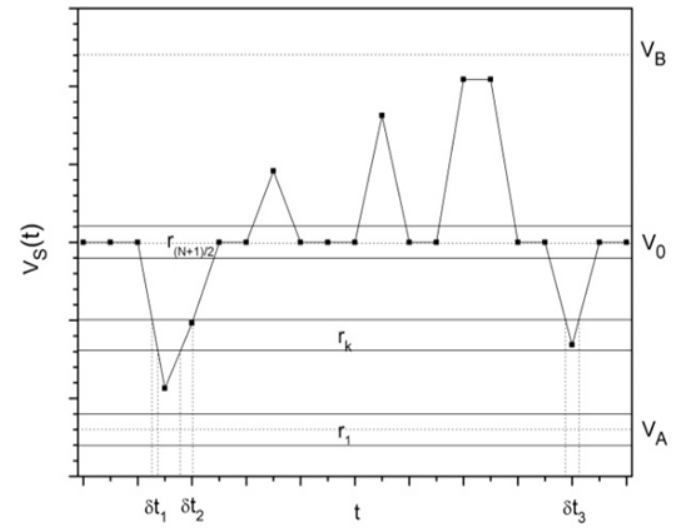

Fig.3 The determination of speed change cumulative distribution function

$$
J_{i}=\frac{V_{r i}}{n \cdot D}, i=1, \cdots, N
$$

The $V_{S}(t)$ is a continuous random variable, and the probability $P\left(V_{S}(t) \in r_{k}\right)$ of actual speed $V_{S}(t)$ distribution in subsets $k$ can be determined according to Fig.3. Actual speed change curve "residence" in the subset within $r_{k}$ is composed of three parts, $\delta t_{1}, \delta t_{2}$ and $\delta t_{3}$. Then, the probability of ship speed distribution can be obtained after the determination of the probability $P\left(V_{S}(t) \in r_{k}\right)=\frac{\delta t_{1}+\delta t_{2}+\delta t_{3}}{T}$ as shown in Table 1 .

Due to the range of $R v$ is relatively small during actual operation of the ship, so when $N$ is large enough, $P\left(V_{S}(t)=V_{r_{k}}\right) \approx P\left(V_{S}(t) \in r_{k}\right)$ is reasonable. Wherein the $V_{r k}$ represents the midpoint of 
$r_{k}$, then the approximate discrete speed distribution law can be obtained as shown in Table 2

$$
\sum_{i=1}^{N} P\left(V_{S}(t) \in r_{i}\right)=1
$$

Table1 The probability distribution of the change speed

\begin{tabular}{cccccc}
\hline$R$ & $r_{1}$ & $\ldots$ & $r_{k}$ & $\ldots$ & $r_{N}$ \\
\hline$P$ & $P\left(v_{S}(t) \in \eta\right)$ & $\ldots$ & $\ldots$ & $\ldots$ & $P\left(v_{S}(t) \in r_{N}\right)$ \\
\hline
\end{tabular}

Table 2 The discipline of speed distribution

\begin{tabular}{cccccc}
\hline$V$ & $V_{r 1}=V_{A}$ & $\ldots$ & $V_{r k}$ & $\ldots$ & $V_{r N}$ \\
\hline$P$ & $P\left(V_{S}(t) \in \eta\right)$ & $\ldots$ & $\ldots$ & $\ldots$ & $P\left(V_{S}(t) \in r_{N}\right)$ \\
\hline
\end{tabular}

Equation (6) can be obtained by equation (4). Therefore, for the optimization model determined by equation (3), the weight value $\omega_{i}$ can be calculated according to the equation (7) when the multi-objective optimization problems are solved by the weighted sum method.

The equation (8) is obviously believable according to equation (5), thus the objective equation can be rebuilt into equation (9).

$$
\begin{aligned}
& f_{i}=\eta_{0}\left(J_{i}\right)=\eta_{O}\left(\frac{V_{r i}}{n D}\right), i=1, \cdots, N \\
& \omega_{i}=P\left(V_{S}(t) \in r_{i}\right), i=1, \cdots, N \\
& \sum_{i=1}^{N} \omega_{i}=1 \\
& z\left(a_{1}, \cdots, a_{6}\right)=\sum_{i=1}^{N} \omega_{i} f_{i}\left(a_{1}, \cdots, a_{6}\right)=\sum_{i=1}^{N} \omega_{i} \eta_{0}\left(J_{i}\right) \\
& P\left(V_{S}(t) \in r_{i}\right)=\int_{r_{i}} f_{V_{S}(t)}\left(v_{S}\right) d v_{S}
\end{aligned}
$$

In addition, for continuous random variable $V_{S}(T)$, if the speed with time curve is not given as shown in Fig.2, but the probability density function $f_{V_{S}(t)}\left(v_{S}\right)$ is available, the probability of actual speed can be calculated by equation (10).

\section{Optimization methods}

Simple genetic algorithm (SGA) was chosen to optimize the calculation in this paper. When the model is solved by SGA algorithm, six variables $a_{1}, a_{2}, a_{3}, \ldots, a_{6}$ are encoded. If cannot meet the constraint conditions, the adaptive value is zero when the individual by adaptive value. Weight and function value are obtained according to equations (9) and the individual is divided by adaptive value according to equation (11), among them $c_{\min }=0.1$.

Fit $(f(x))=\left\{\begin{array}{cc}f(x)-c_{\min } & f(x)>c_{\min } \\ 0 & \text { other }\end{array}\right.$

Wherein, $c_{\min }$ is the minimum estimation of the objective function.

Others operation parameters are set as follow.

The population size $M=50$. The biggest evolution algebra $T=20$. The crossover probability $P C$ = 0.9. The mutation probability $\mathrm{Pm}=0.1$.

\begin{tabular}{|c|c|c|c|c|}
\hline $0.18 R$ & pitch ratio & pitch ratio of tip & $0.75 R$ pitch ratio & $0.7 R$ pitch ratio \\
\hline & 1.3 & 0.9 & 1.02 & 1.05 \\
\hline
\end{tabular}

\section{Optimization example and results analysis}

\section{Optimization example}

The AU5-65 propeller is used as the parent form, and the initial value of pitch ratio curve of radial distribution $P(r)_{0} / D$ is set according to the plan in Table 3.

Table 3 The initial pitch ratio distribution 
For this multi-objective, $J_{0}=0.86$ is advance point of design, field radius is $\Delta=0.05$, therefore $J_{A}=0.81, J_{B}=0.91$. Take the number of objective function as 5 . The five objective functions are shown as equation (13).

$$
\begin{aligned}
& f_{1}=\eta_{O}(J=0.81) \\
& f_{2}=\eta_{O}(J=0.835) \\
& f_{3}=\eta_{O}(J=0.86) \\
& f_{4}=\eta_{o}(J=0.885) \\
& f_{5}=\eta_{O}(J=0.91)
\end{aligned}
$$

Because the exact speed $V_{S}(t)$ changes with time and the probability density function after statistical analysis are unavailable in this paper. For this example, assuming the change of the actual speed $V_{S}(t)$ obey the normal distribution because of the influence of wind, wave and flow leads to, namely $V_{S}(t) \sim N\left(\mu, \sigma^{2}\right)$. $\mu$ is the location parameter, namely, it is the distribution of expectations. $\sigma$ is the scale parameter, namely it is the standard deviation of the distribution, which size can express the random variable $V_{S}(t)$ concentration distribution near the expectations $V_{0}$. $\sigma$ is more smaller, the concentration is more higher.

In this example, $\mu=V_{0}=n \cdot D \cdot J_{0}, \sigma$ can be calculated by the equation (14).

$$
8 \square \sigma=N\left(\frac{V_{B}-V_{A}}{N-1}\right)
$$

Wherein, $N=5, V_{A}=n \cdot D \cdot J_{A}, V_{B}=n \cdot D \cdot J_{B}$. The equation said $\left[V_{0}-4 \sigma, V_{0}+4 \sigma\right]$ are average divided into $N$ subsets $r_{i}, i=1, \cdots, N$, and to guarantee the halfway point of the first subset $r_{1}$ is $V_{A}$, the halfway point of the $N$ subset $r_{N}$ is $V_{B}$. The five classified subsets $r_{i}, i=1, \cdots, 5$ and its interval endpoint are shown in Table 4.

Table 4 The partition of subset

\begin{tabular}{cccc}
\hline sunset & range & midpoint & advance coefficient \\
\hline$\left[v_{0}-4 \sigma, V_{0}-2.4 \sigma\right]$ & {$[1.99,2.06]$} & 2.025 & 0.81 \\
{$\left[V_{0}-2.4 \sigma, V_{0}-0.8 \sigma\right]$} & {$[2.06,2.12]$} & 2.087 & 0.835 \\
{$\left[V_{0}-0.8 \sigma, V_{0}+0.8 \sigma\right]$} & {$[2.12,2.18]$} & 2.15 & 0.86 \\
{$\left[V_{0}+0.8 \sigma, V_{0}+2.4 \sigma\right]$} & {$[2.18,2.24]$} & 2.212 & 0.885 \\
{$\left[V_{0}+2.4 \sigma, V_{0}+4 \sigma\right]$} & {$[2.24,2.31]$} & 2.275 & 0.91 \\
\hline
\end{tabular}

Table 4 shows that the advance coefficient of halfway point of subset interval is same as the advance coefficient value of five objective functions.

And the known normal distribution density function is equation (15).

$$
f(x ; \mu, \sigma)=\frac{1}{\sigma \sqrt{2 \pi}} \exp \left(-\frac{(x-\mu)^{2}}{2 \sigma^{2}}\right)
$$

According to the standard normal distribution table, cumulative interval distribution value is shown in Table 5. According to Table 5 the speed change distribution probability of Table 1 can be calculated as shown in Table 6. Finally, the weight value of five objective functions is shown in Table 7 in this example.

Finally the objective function can be expressed as equation (16).

Table 5 Interval cumulative distribution value

\begin{tabular}{cccccccc}
\hline$x$ & & 2.00 & 2.06 & 2.12 & 2.18 & 2.24 & 2.31 \\
\hline$P(x \leq x)$ & & 0 & 0.01 & 0.21 & 0.79 & 0.99 & 1 \\
\hline$R$ & \multicolumn{7}{c}{ Table 6 The probability distribution of the change speed } \\
\hline$P$ & & $r 1$ & $r 2$ & $r 3$ & $r 4$ & $r 5$ \\
\hline
\end{tabular}


Table 7 The weights

\begin{tabular}{cccccc}
\hline$\omega_{\mathrm{i}}$ & $\omega_{1}$ & $\omega_{2}$ & $\omega_{3}$ & $\omega_{4}$ & $\omega_{5}$ \\
\hline values & 0.0082 & 0.2037 & 0.5762 & 0.2037 & 0.0082 \\
\hline
\end{tabular}

$z\left(a_{1}, \cdots, a_{6}\right)=0.0082 \cdot f_{1}+0.2037 \cdot f_{2}+0.5762 \cdot f_{3}+0.2037 \cdot f_{4}+0.0082 \cdot f_{5}$

\section{Analysis results}

According to equation (14) the objective function value can be calculated and the initial pitch curve can be determined, therefore the model can be optimized based on SGA algorithm according to the optimization process of Fig.4. Variations of genetic optimization objective function are shown in Fig.4.

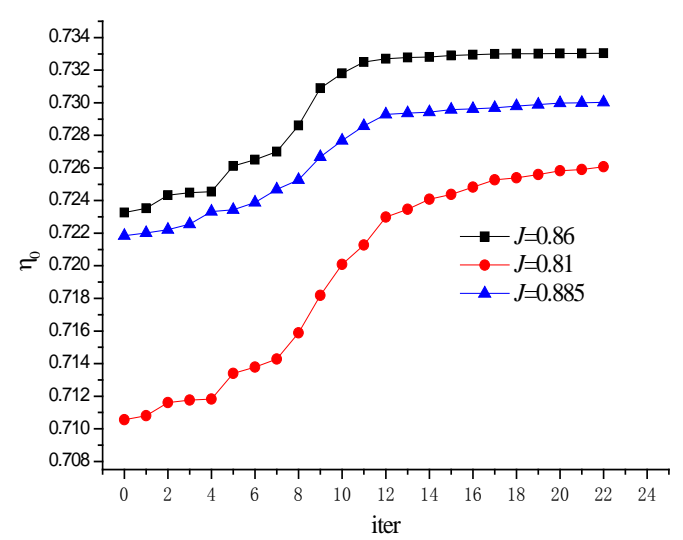

Fig.4 Variation curves of objective function.

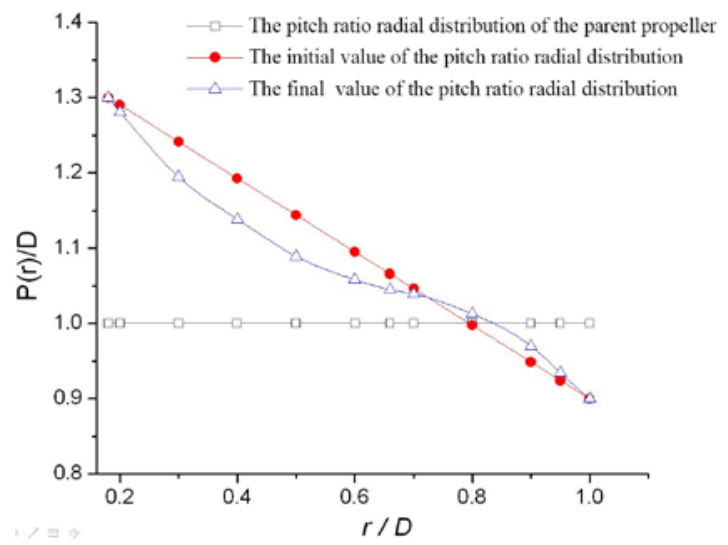

Fig.5 The radial distribution of the pitch ratio before and after optimizing

The optimized radial distribution curves of pitch ratio are shown in Fig. 5. The radial distribution curve optimized pitch ratio remains generally monotone decrease by contrast with the final result and the change rate of propeller on the root and tip is bigger than central blade, pitch ratio changes in central relatively flat.

Table 8 shows open water efficiency of original propeller only considered the design advance coefficient, and the multi-objective optimization design results. It can be seen that single objective optimization and multi-objective optimization can improve open water efficiency in $J=0.86$. Compared with the original propeller, single objective optimization has increased by $0.62 \%$, while the multi-objective optimization has improved by $0.986 \%$.

Table 8 The comparison of open water efficiency under different advance coefficients

\begin{tabular}{|c|c|c|c|c|}
\hline$J$ & Initial pro $\eta_{0}$ & Single-objective $\eta_{0}$ & multi-objective $\eta_{0}$ & weight \\
\hline 0.81 & 0.71055 & 0.70265 & 0.71281 & 0.0082 \\
\hline 0.835 & 0.71846 & 0.71921 & 0.72482 & 0.2037 \\
\hline 0.86 & 0.72327 & 0.73228 & 0.73206 & 0.5762 \\
\hline 0.885 & 0.72185 & 0.72116 & 0.72538 & 0.2037 \\
\hline 0.91 & 0.71015 & 0.70626 & 0.71274 & 0.0082 \\
\hline sum & 0.72179 & 0.72628 & 0.72891 & \\
\hline
\end{tabular}

\section{Conclusions}

In this paper, based on the simple genetic algorithm combined with the weighted sum method, the optimization method considered off-design points efficiency is established. Taking AU5-65 atlas as original propeller, optimization design is carried out. The comparison results show that,

1) Whether considering the efficiency of design point or the efficiency within the scope of the point of design conditions, the propeller efficiency has improved in some extent compared with the original map, which illustrates that propeller performance of atlas design exist optimization space.

2) Optimizing by considering the efficiency within the scope of design conditions, the weight values play a decisive role in the optimized effect, while the weight values depend on the statistical 
results of ship speed distribution in the process of operation.

3) If only the efficiency of the design point is considered, the optimization effect in the process of actual operation will be significantly lower, while the optimization effect is better by considering the efficiency within the scope of design conditions.

The work of this paper tries to optimize propeller design considered off-design conditions. The purpose is to establish a new optimization idea and method. Only radial distribution of pitch ratio is selected as the design variables and the optimization goal also only choose the efficiency. The further step is to study multivariate multi-objective optimal design problems.

\section{Acknowledgements}

This study was supported by grants from the National Natural Science Foundation of China (Grant NO. 51379040).

\section{References}

[1] Wei Zhao, ChenJun Yang. Research on Optimized Design of the Pitch and Camber of Marine Propellers [J] Shipbuilding of China, 2010,01, p.1-8.

[2] Zhibo Zeng, Enbao Ding, Denghai TANG. Ship propeller design optimization based on BP neural network and genetic algorithm [J]. Journal of Ship Mechanics,2010,Z1, p.20-27.

[3] Chunyu Guo, Chao Wang. Application and Comparison of Two Different Optimization Algorithms in the Optimization Design of Propeller [J] Ship Engineering,2013,03:p.23-26.

[4] Peng Wang, Shuai Huang. Application of Swarm Intelligence Optimization Algorithm in Parameter Optimization Design of Propeller. Computer Science [J],2013,S2:73-76.

[5] Xin Chang, Chunyu Guo, Xiangyin Meng etal. Design of Hydrofoil Section based on Particle Swarm Optimization[J]. Ship Engineering,2010,05:1-3.

[6] Ping $\mathrm{Xu}$, Changsheng Jiang. Optimization Design of Laminar Aerofoil Based on Genetic Algorithm sand Hicks-Henne Shape Function [J] Journal of Air force Engineering University, 2009, 10(1)

[7] Zhenbang Sheng, Yingzhong Liu. The principle of ship[M]. Shanghai Jiao Tong University press.2004.77-80. 